\title{
ANÁLISIS BIBLIOMÉTRICO SOBRE EL BIENESTAR SUBJETIVO
}

\author{
Gerardo Riffo Allende \\ Universidad Pedro de Valdivia, Chile \\ gerardo.riffo@upv.cl \\ Andrea Donoso Aguirre \\ ONG Agencia Adventista de Desarrollo y \\ Recursos Asistenciales (ADRA-Chile) \\ psi.andreadonoso@gmail.com
}

Fecha de Recepción: 6 Agosto 2019

Fecha de Admisión: 25 Septiembre 2019

\section{RESUMEN}

El objetivo de este estudio ex post facto retrospectivo es describir el bienestar subjetivo mediante un análisis bibliométrico del periodo 1998-2018 a partir de las bases de datos Scopus. Durante dicho periodo se han publicado 2450 artículos en dicha base de datos.

Se han analizado la productividad y distribución temporal, así como la producción científica por países, idiomas, autores, revistas, universidades y organismos financiadores, identificando aquellos más productivos.

Los principales resultados encontrados hacen referencia a un aumento de la producción científica a nivel mundial sobre el bienestar subjetivo principalmente entre los años 2013 a 2018, asimismo existe un predominio de las publicaciones en idioma inglés, donde los países más productores son Estados Unidos, Alemania y Reino Unido y los principales referentes en producción científica son Diener, Ferran y Oichi.

Estos resultados, a su vez, podrán utilizarse como fundamento para posteriores proyectos de investigación que aporten al progreso de la ciencia.

Palabras clave: análisis bibliométrico; bienestar subjetivo; estudio ex post facto retrospectivo; productividad científica.

\section{ABSTRACT}

Bibliometric analysis of subjective well-being. The objective of this retrospective ex post facto study is to describe the subjective well-being by means of a bibliometric analysis of the 1998-2018 time period using the Scopus database. During this period, 2450 articles have been published in this database.

Not only have been productivity and temporal range analyzed, but also scientific production rates by countries, languages, authors, journals, universities and supporting organisms, identifying the highest productivity rates.

The main results found refer to an increase in global scientific production on subjective well-being mainly between 2013 and 2018, there is also a predominance of publications in English language, where the most pro- 


\section{ANÁLISIS BIBLIOMÉTRICO SOBRE EL BIENESTAR SUBJETIVO}

ducing countries are the United States, Germany and the United Kingdom and the main references in scientific production are Diener, Ferran and Oichi.

These results, in turn, will be able to be in use as base for successive research projects that contribute to the progress of the science.

Keywords: bibliometric analysis; subjective well-being; retrospective ex post facto study; scientific productivity.

\section{INTRODUCCION}

Durante décadas la disciplina psicológica se ha enfocado en el estudio y abordaje de las patologías mentales, generando teorías para una comprensión sobre el funcionamiento humano y el desarrollo de intervenciones y terapias para las diferentes alteraciones o enfermedades, permitiendo de esta forma un avance en la recuperación de las personas, familias, grupos o comunidades.

Es así, que durante gran parte de su historia, la psicología se ha despreocupado de los aspectos positivos del ser humano, no considerándolos como objetos de estudio o bien desconociendo los beneficios que otorgan a las personas, familias o grupos, tales como, la satisfacción con la vida, los valores, el coraje, la esperanza, el optimismo, la felicidad, el flujo, el bienestar, entre otros (Seligman \& Csikszentmihalyi, 2000). Dentro de ellas, en las últimos años ha tomado gran relevancia el tema del bienestar por su campo de acción e investigación en diferentes disciplinas y áreas, lo que la llevo incluso a posicionarla como un indicador a nivel mundial (Alfaro, Casas \& López, 2015; Muñoz Umaña, 2007).

El concepto de bienestar subjetivo (subjective well-being en inglés) es entendido como un constructo psicológico que expresa el nivel en que las personas creen (componente cognitivo) y sienten (componente afectivo) que sus vidas son gratificantes y amenas y que es evaluada de forma favorable o positiva cuando existe consonancia entre la propia visión y la calidad de vida percibida (Diener 1984; Diener \& Larsen, 2009; Diener, Scollon \& Lucas, 2009; Pavot \& Diener, 2009). Uno de sus principales exponente Ed. Diener (1994) refiere que este concepto tiene tres elementos a destacar, los que son la satisfacción con la vida, las experiencias constantes de afectos positivos y la ausencia de afectos negativos.

Desde esta configuración, la persona utiliza dos elementos en este análisis, por un lado esta el componente cognitivo, que incorpora la discrepancia percibida entre sus aspiraciones y sus logros, cuyo rango de análisis va desde la sensación de una realización personal plena hasta la experiencia vital de fracaso o decepción, lo cual es denominado como satisfacción con la vida. Por otra parte, está el componente afectivo, que constituye el plano hedónico, lo que refiere al agrado experimentado por el propio sujeto con sus emociones, sentimientos y estados de ánimo más habituales (Lucas, Diener y Suh, 1996; Pavot \& Diener, 2009; Rodriguez-Fernandez \& GoniGrandmontagne, 2011; Vazquez, Duque \& Hervas, 2013).

Dentro de los múltiples aspectos favorables que tiene el bienestar subjetivo, Seligson, Huebner y Valois (2003) establecen cuatro aspectos favorecedores de la salud: la integración de perspectivas psicológicas y sociológicas con la perspectiva médica; la inclusión de factores personales, comunitarios y estructurales como elementos determinantes en la salud; la implementación de ambientes que fomenten comportamientos saludables orientados a la promoción y rehabilitación de salud; y el desarrollo de test y pruebas de carácter psicométricos que permitan evaluar las intervenciones y abordajes realizados en el ámbito de la salud. Todos estos elementos son reconocidos y esenciales para la promoción de la salud a nivel biopsicosocial y también para el desarrollo de una mejor calidad de vida (Cuadra \& Florenzano, 2003; Diener, 2000; Diener, 2006; Diener, Suh, Lucas \& Smith, 1999).

Cabe destacar que este concepto, en las últimas décadas, ya no solo se aborda desde la psicología, sino que presenta investigación empírica y teórica desde otras disciplinas, entre las que destacan la economía, la ciencia política, el trabajo social y la sociología, fundamentalmente orientada al desarrollo y aumento del bienestar y de la calidad de vida en la sociedad. Un dato relevante, es que en el campo de las políticas públicas Bután en los 
años setenta crea el Ministerio de la Felicidad y realiza un proceso innovador que es la medición de la Felicidad Nacional Bruta, desde allí, numerosos países del mundo han incorporado el bienestar subjetivo como un elemento esencial y prioritario en sus políticas y procesos (Cummins, Eckersley, Van Pallant, Vugt \& Misajon, 2003; Peñaguirre Cano \& Rodríguez Mendoza, 2017).

La importancia que ha adquirido en los últimos años el estudio del bienestar subjetivo en el mundo se aprecia en la incorporación de esta temática en múltiples organismos interestatales, es así que el año 2008, el gobierno francés genera una comisión de análisis que elabora el Informe de la Comisión sobre la Medición del Desarrollo Económico y del Progreso Social y el 19 de julio de 2011 las Naciones Unidas emiten la resolución 65/309 que insta a que los países puedan generar políticas públicas orientadas al desarrollo de la felicidad y el bienestar. El mismo año la Organización de Cooperación y Desarrollo Económico (OCDE) genera el índice para una Vida Mejor (Your Better Life Index) el cual tiene 11 dimensiones, donde destaca la satisfacción ante la vida (Programa de las Naciones Unidas para el Desarrollo, 2012).

Por otra parte, también es posible evidenciar los nuevos centros e institutos a nivel mundial que han aparecido en los últimos años, como el Centre for Well-being en Inglaterra que genera el Happy Planet Index, el World Database of Happiness desarrollado por Ruth Veenhoven en los Países Bajos y en Latinoamérica a través de las investigaciones y reportes del Latinobarómetro (Farías, Orellana \& Pérez, 2015; Rojas, 2011).

Como es posible visualizar, dicho concepto está adquiriendo énfasis a nivel mundial, aumentando el número de investigaciones que lo abordan de manera sustancial en el último periodo, por lo que parece relevante, conocer el estado del arte del bienestar subjetivo y revisar el status de conocimiento de las actuales investigaciones publicadas en esta temática, considerando que, los análisis de tipo bibliométricos son una herramienta que permite conocer la producción científica y profundizar en el impacto que tiene a nivel social (Rueda-Clausen, Villaroel Gutierrez \& Rueda-Clausen, 2005).

\section{METODO}

Según la clasificación de Montero y León (2007), se trata de un estudio ex post facto retrospectivo, en el que se presentan los resultados de un análisis bibliométrico realizado sobre el bienestar subjetivo durante el periodo 1998-2018. Se escoge este año de inicio, ya que, Seligman brinda las bases del surgimiento de la psicología positiva, proceso que lleva a potenciar el estudio de esta y otras variables más.

La bibliometría se entiende como un compilado de técnicas y procedimientos para estudiar la naturaleza y comportamiento de una disciplina mediante la sistematización y análisis de los diversos aspectos de la comunicación escrita, teniendo en cuenta una metodología específica que se ocupa del análisis cuantitativo de determinadas variables presentes en un texto publicado (Pérez et al., 2003).

Se ha utilizado la base de datos SCOPUS para el presente estudio, esto ya que mantiene una posición relevante entre las bases de datos bibliográficas (Andres, 2009). Cabe mencionar que las revistas incluidas en ella presentan estándares muy altos de calidad, y esta base facilita la recuperación de información y datos relevantes para este tipo de estudios.

Se llevó a cabo una búsqueda de la expresión Subjective Well-Being dentro del campo keywords (conceptos clave), se uso el término en inglés por ser el idioma de uso común en SCOPUS. La selección de este concepto fue con la intención de obtener una muestra, lo más amplia posible, de publicaciones relacionadas al concepto escogido. Sumado a ello, se refino la búsqueda usando los siguientes filtros: 1) Year: 1998 a 2018; 2) Document Type: Article; 3) Publication Stage: Final; y 4) Source Type: Journal, dando un total de 2450 documentos para llevar a cabo el proceso de investigación.

En el análisis se incluye el número de artículos publicados, los autores más productivos, los países más productores, las universidades de afiliación más productoras, las revistas especializadas, las áreas de investigación, el tipo de estudio y la evolución temporal de los trabajos publicados durante este periodo. Una vez organizada la información, se efectuó la aplicación del análisis estadístico de las variables que lo permitían para lograr obtener los datos más relevantes sobre el estado de la investigación en el tema. 


\section{ANÁLISIS BIBLIOMÉTRICO SOBRE EL BIENESTAR SUBJETIVO}

\section{RESULTADOS}

Como resultado de la búsqueda bibliográfica se hallaron 2450 artículos científicos en el período comprendido entre 1998 y 2018, teniendo en cuenta los criterios de inclusión y exclusión establecidos previamente.

\section{Tipo de acceso de la publicación:}

Como se puede observar en la figura 1, de los 2450 artículos científicos referidos al bienestar subjetivo, podemos encontrar 376 artículos de acceso libre (Open Access) lo que corresponde a un 15,3\% del total revisado.

Figura 1. Tipo de acceso de las publicaciones

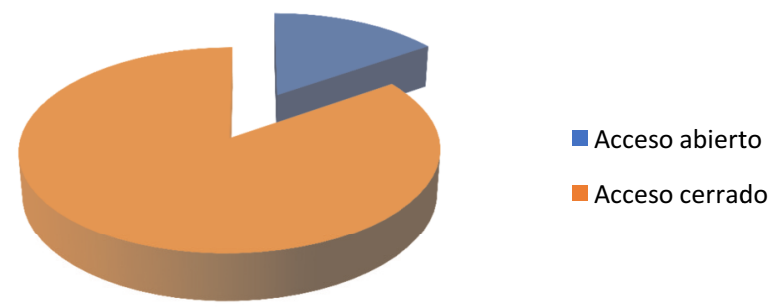

\section{Productividad temporal}

El número de artículos científicos que incluyen el término bienestar subjetivo ha crecido de manera importante con el paso de los años. En el periodo comprendido entre 1998 y 2008 se publicaron 317 artículos, lo que supone un 12,9\% del total analizado. En los últimos 5 años se evidencia un crecimiento e interés fuerte en este tema, ya que se han publicado 1377 , lo que corresponde a un $56.2 \%$ de lo revisado.

Figura 2. Evolución temporal de los trabajos publicados

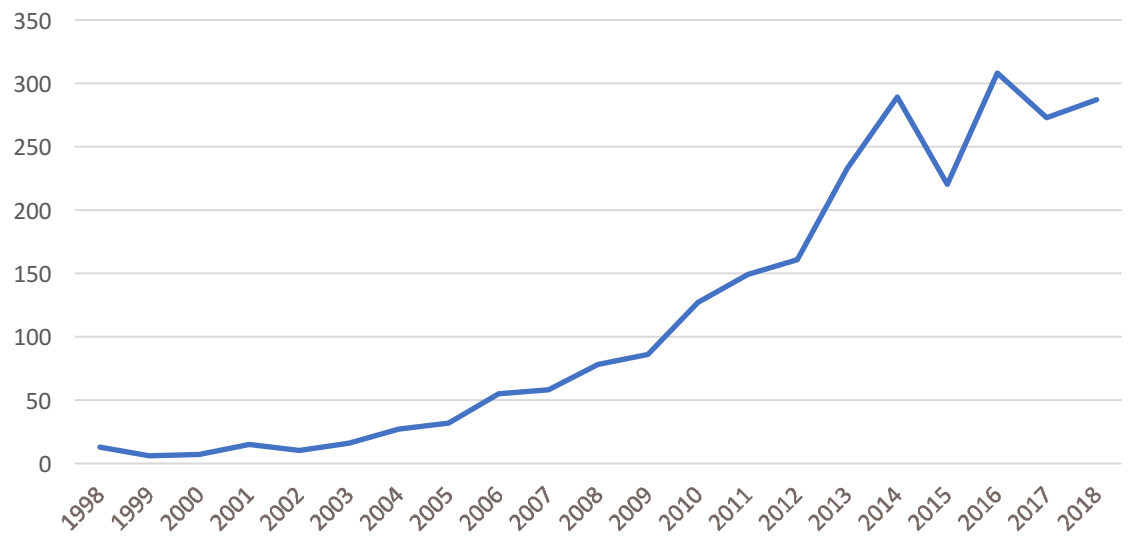




\section{Productividad de autores y de países}

La tabla 1 nos muestra a los 15 autores que mas artículos científicos han publicado sobre el Bienestar subjetivo entre 1998 y 2018. Podemos observar que el autor más productor es Ed Diener con 37 artículos, seguido de cerca por Ferran Casas con 32 artículos y Shigehiro Oichi con 24.

También se ha realizado una distribución de los artículos publicados en función de los países más productores. En la tabla 2 podemos observar como las publicaciones de Estados Unidos recogen el mayor número de artículos científicos (654) sobre el bienestar subjetivo con una importante diferencia respecto al resto de los países del mundo. De hecho, el segundo país que más produce es Alemania con 266 artículos seguido de Reino Unido con 221 publicaciones.

Tabla 1. Autores más productivos

\begin{tabular}{cc}
\hline Autor & $\mathbf{N}^{\circ}$ de Artículos \\
\hline Ed Diener & 37 \\
\hline Ferran Casas & 32 \\
\hline Shigehiro Oichi & 24 \\
\hline Richard E. Lucas & 20 \\
\hline Louis Tay & 16 \\
\hline Martin Binder & 15 \\
\hline Mohsen Joshanloo & 13 \\
\hline Maike Luhmann & 13 \\
\hline Heinz Welsch & 13 \\
\hline Sonja Lyubomirsky & 11 \\
\hline Danilo García & 10 \\
\hline Corey Keyes & 10 \\
\hline Michael Lambert & 10 \\
\hline Francesco Sarracino & 10 \\
\hline Alois Stutzer & 10
\end{tabular}

Tabla 2. Países productores

\begin{tabular}{ccc}
\hline Países productores & $\mathbf{N}^{\circ}$ de Artículos & Porcentaje \\
\hline Estados Unidos & 654 & $\mathbf{2 6 . 7}$ \\
\hline Alemania & 266 & $\mathbf{1 0 . 9}$ \\
\hline Reino Unido & 221 & $\mathbf{9 . 0}$ \\
\hline China & 203 & $\mathbf{8 . 3}$ \\
\hline España & 146 & $\mathbf{6 . 0}$ \\
\hline Canadá & 137 & $\mathbf{5 . 6}$ \\
\hline Australia & 116 & $\mathbf{4 . 7}$ \\
\hline Países bajos & 96 & $\mathbf{3 . 9}$ \\
\hline Corea del Sur & 84 & $\mathbf{3 . 4}$ \\
\hline Japón & 78 & $\mathbf{3 . 2}$
\end{tabular}




\section{Idioma}

Como se puede observar en la tabla 3, existen mayoritariamente publicaciones sobre el bienestar subjetivo en el idioma inglés (2248) representando más del 90\% de todos los artículos analizados, lo que se relaciona directamente con que el país que más pública sobre este tema es Estados Unidos y que el idioma empleado por la ciencia por excelencia es el inglés. Muy por debajo, existen otros idiomas como el Español con 55 artículos, seguido del Portugués (34), Alemán (27) y Ruso (25).

Tabla 3. Idioma de la publicación

\begin{tabular}{ccc}
\hline Idioma & $\mathbf{N}^{\circ}$ de Artículos & Porcentaje \\
\hline Ingles & 2248 & 91.8 \\
\hline Español & 55 & 2.2 \\
\hline Portugués & 34 & 1.4 \\
\hline Alemán & 27 & 1.1 \\
\hline Ruso & 25 & 1.0 \\
\hline Otros (18) & 61 & 2.5 \\
\hline
\end{tabular}

\section{Revistas y Universidades especializadas}

Se han analizado las diferentes revistas especializadas que han publicado artículos relacionados a este tema, entre ellas destacan dos que son Social Indicators Research con un 10,2\%, seguida de Journal Of Happiness Studies con un 7,4\%. En la tabla 4 se recogen las 20 principales revistas que han publicado trabajos sobre el bienestar subjetivo entre los años 1998 a 2018.

Tabla 4. Distribución de artículos en revistas científicas

\begin{tabular}{lcc}
\hline \multicolumn{1}{c}{ Revista } & $\begin{array}{c}\mathbf{N}^{\circ} \text { de } \\
\text { Artículos }\end{array}$ & \% \\
\hline Social Indicators Research & 251 & 10.2 \\
\hline Journal Of Happiness Studies & 181 & 7.4 \\
\hline Personality And Individual Differences & 63 & 2.6 \\
\hline Journal Of Positive Psychology & 38 & 1.6 \\
\hline Child Indicators Research & 36 & 1.5 \\
\hline Applied Research In Quality Of Life & 27 & 1.1 \\
\hline Frontiers In Psychology & 27 & 1.1 \\
\hline Journal Of Economic Behavior And & & \\
Organization & 27 & 1.1 \\
\hline Journal Of Research In Personality & 25 & 1.0 \\
\hline Ecological Economics & 20 & 0.8 \\
\hline Quality Of Life Research & 20 & 0.8 \\
\hline Children And Youth Services Review & 18 & 0.7 \\
\hline Journal Of Economic Psychology & 18 & 0.7 \\
\hline Journal Of Socio Economics & 18 & 0.7 \\
\hline Social Behavior And Personality & 18 & 0.7 \\
\hline Computers In Human Behavior & 17 & 0.7 \\
\hline Journal Of Personality And Social & & \\
Psychology & 17 & 0.7 \\
\hline Aging And Mental Health & 14 & 0.6 \\
\hline Journal Of Cross Cultural Psychology & 14 & 0.6 \\
\hline Journals Of Gerontology Series B & & \\
Psychological Sciences And Social & & \\
Sciences & 14 & 0.6 \\
\hline
\end{tabular}


En la tabla 5 se pueden observar las 20 Universidades que principalmente han publicado artículos científicos en este campo. La Universitat de Girona (España) es la institución que mas publicaciones ha realizado sobre Bienestar subjetivo de 1998 a 2018 con 37 artículos, seguida de la University of Illinois at Urbana-Champaign (Estados Unidos) con 29 artículos y la Göteborgs Universitet (Suecia) con 28 artículos.

La mayoría de las principales instituciones universitarias que aparecen son norteamericanas (7) 0 europeas (7) y en menor cantidad en otras regiones geográficas como Asia (3), Oceanía (2) y América del Sur (1).

Tabla 5. Distribución de artículos según Universidad

\begin{tabular}{lll} 
Afiliación & $\begin{array}{c}\mathbf{N}^{\circ} \mathbf{d e} \\
\text { publicaciones }\end{array}$ & $\mathbf{\%}$ \\
\hline Universitat de Girona & 37 & 1.5 \\
\hline University of Illinois at Urbana-Champaign & 29 & 1.2 \\
\hline Göteborgs Universitet & 28 & 1.1 \\
\hline University of Virginia & 27 & 1.1 \\
\hline The University of British Columbia & 23 & 0.9 \\
\hline University of Michigan, Ann Arbor & 23 & 0.9 \\
\hline University of Zurich & 22 & 0.9 \\
\hline Universitätsklinikum Hamburg-Eppendorf & & \\
und Medizinische Fakultät & 21 & 0.9 \\
\hline University of Missouri-Columbia & 21 & 0.9 \\
\hline Monash University & 21 & 0.9 \\
\hline Erasmus University Rotterdam & 21 & 0.9 \\
\hline Michigan State University & 21 & 0.9 \\
\hline Beijing Normal University & 20 & 0.8 \\
\hline University of Manchester & 19 & 0.8 \\
\hline Universidade Federal do Rio Grande do Sul & 19 & 0.8 \\
\hline Institut Zur Zukunft Der Arbeit & 19 & 0.8 \\
\hline University of Melbourne & 19 & 0.8 \\
\hline University of Haifa & 18 & 0.7 \\
\hline Purdue University & 18 & 0.7 \\
\hline Chinese University of Hong Kong & 17 & 0.7 \\
\hline
\end{tabular}

\section{CONCLUSIONES}

El objetivo de este trabajo ha sido realizar una revisión bibliométrica durante el periodo de 1998 y 2018 sobre la temática de Bienestar Subjetivo a partir de la base de datos Scopus. Durante el transcurso temporal revisado, se identificaron 2450 artículos científicos y a partir del año 2013 se produce un incremento significativo de la producción científica en este tema, alcanzando el nivel más alto de productividad el año 2016 con 308 publicaciones, esto permite reflejar una evolución ascendente demostrando que el bienestar subjetivo es un tópico de relevancia actual y en continuo y permanente desarrollo.

Respecto a las publicaciones, se evidencia que las publicaciones de acceso abierto aun son bajas e incipientes a pesar que desde el año 2001 se origino el movimiento Open Access con el objetivo de potenciar el acceso abierto a la literatura científica, es así que solo un poco más del 15\% de los artículos analizados pueden accederse sin barreras legales, técnicas y/o financieras. 


\section{ANÁLISIS BIBLIOMÉTRICO SOBRE EL BIENESTAR SUBJETIVO}

En la base de datos SCOPUS podemos revisar que las publicaciones relacionadas al bienestar subjetivo están en más de 20 idiomas distintos, pero casi el 92\% de las publicaciones se realiza en inglés, entendiéndolo como el idioma científico por excelencia. Esto explica que el país más productor sea EEUU con una amplia diferencia respecto al resto y en tercer lugar se encuentre el Reino Unido. Por otra parte, países no angloparlantes como Alemania, España o China se encuentran dentro de las 5 naciones más productoras y el nivel de idioma nativo es bajo (menos del $5 \%$ sumándolos) hace pensar que la gran mayoría de sus aportaciones las realizan en inglés, entendiendo que esto les permite una mayor visibilidad y posicionamiento o bien las principales revistas fomentan este idioma para facilitar su indexación en bases internacionales.

En relación a la productividad de los autores, se vislumbra que la mayor producción se concentra en tres autores, Ed. Diener, Ferran Casas y Shigehiro Oichi, quienes hasta la fecha son considerados importantes exponentes del bienestar a nivel mundial. Estos autores tienen sus grupos de investigación en Universidades norteamericanas y europeas, zonas con alto nivel de productividad en este campo.

En cuanto a las revistas, se considera un diverso número de revistas especializadas en este ámbito ya que durante el periodo revisado la editorial con mayor difusión de artículos sobre bienestar subjetivo es Social Indicators Research con más de 250 artículos, dicha fuente junto con las revistas Journal of Happiness Studies y Personality and Individual Differences, son en las que más se publica sobre esta temática siendo las principales fuentes de difusión científica.

Este análisis se espera que pueda ser una referencia para futuros estudios y permita proyectar las investigaciones teniendo en cuenta el desarrollo y auge de la psicología positiva y de uno de sus conceptos centrales como es el bienestar subjetivo. Por esto, los resultados presentados en este estudio pueden tomarse como un punto de inicio para identificar las principales tendencias de investigación y así potenciar el análisis conceptual vinculado a esta temática.

Los indicadores de tipo bibliométricos actualmente constituyen una de las herramientas más utilizadas para la medición del producto de la investigación científica, ya que estos proporcionan datos sobre una determinada temática o proceso. Por esto mismo, en numerosos países estos indicadores se han consolidado como instrumentos de apoyo para la producción científica permitiendo identificar las tendencias en investigación y en qué medida estas responden a las necesidades de un país o de una región específica del mundo.

Dentro de las limitaciones que presenta el estudio, se considera que no analiza ni da cuenta del índice de colaboración entre países, ya que existen revistas que dan cabida a trabajos nacionales, mientras que para otras revistas el criterio a utilizar consiste en obtener un mayor número de colaboradores extranjeros, por otro lado, tampoco se considera un análisis de colaboración, por lo que no nos permite saber si las investigaciones fueron firmadas por dos o más autores y como es la tendencia de ellos en el periodo analizado o bien entender si existen equipos de trabajo en esta área o si son esfuerzos y trabajos autónomos de algunos exponentes.

Por otra parte, puede existir un sesgo de publicación debido a que sólo se trabajó con artículos publicados y es posible que existan trabajos relacionados al bienestar subjetivo que aún no hayan sido publicados, por lo cual, los datos pueden no reflejar en forma totalmente fiel el "estado actual de la cuestión", justamente debido a que muchos artículos pueden encontrarse en proceso de revisión o en prensa.

Para concluir, se puede afirmar que, a pesar de las diversas limitaciones, el presente análisis bibliométrico constituye un trabajo de gran importancia ya que contribuye al conocimiento de la actividad científica referida a uno de los pilares centrales de la psicología positiva, que es el Bienestar subjetivo, sistematizando datos relacionados a este tema, evaluándolos cuantitativamente e identificando las tendencias actuales en este campo de estudio.

\section{REFERENCIAS BIBLIOGRÁFICAS}

Alfaro, J., Casas, F. \& López, V. (2015). Bienestar en la infancia y adolescencia. Psicoperspectivas: Individuo y Sociedad, 14(1), 1-5. 
Andrés, A. (2009). Measuring academic research. How to undertake a bibliometric study. Cambridge: Chandos Publishing.

Cuadra, H. \& Florenzano, R. (2003). El bienestar subjetivo: hacia una psicología positiva. Revista de Psicología de la Universidad de Chile, 12(1), 83-96.

Cummins, R.A., Eckersley, R., Van Pallant, J., Vugt, J. y Misajon, R. (2003). Developing a national index of subjective well-being: The Australian unity well-being index. Social Indicators Research, 64, 159-190.

Diener, E. (1984). Subjective well-being. Psychological Bulletin, 95, 542-575.

Diener, E. (1994). El bienestar subjetivo. Intervención psicosocial, 3, 67-113.

Diener, E. (2000). Subjective well-being: The science of happiness and a proposal for a national index. American Psychologist. 55; $34-43$.

Diener, E. (2006). Guidelines for national indicators of subjective well-being and ill-being. Applied Research in Quality of Life, 1, 151-157.

Diener, E. \& Larsen, R.J. (2009). Temporal stability and cross-situational consistency of affective, behavioral, and cognitive responses. En E. Diener (Ed.) Assessing well-being. The collected works of Ed Diener (pp. 7-24). Nueva York: Springer (Social Indicators Research Series, 39).

Diener, E., Scollon, C.N. \& Lucas, R.E. (2009). The evolving concept of subjective well-being: The multifaceted nature of happiness. En E. Diener (Ed.) Assessing well-being. The collected works of Ed Diener (pp. 67-100). Nueva York: Springer (Social Indicators Research Series, 39).

Diener, E., Suh, E., Lucas, R. \& Smith, H. (1999). Subjective well-being: Three decades of progress. Psychological Bulletin, 125, 276-302.

Farías, F., Orellana, C. \& Pérez, C. (2015). Perfil de las Publicaciones sobre Bienestar Subjetivo en Chile. Cinta de moebio, 54, 240-249.

Lucas, R.E., Diener, E. \& Suh, E. (1996). Discriminant validity of wellbeing measures. Journal of Personality and Social Psychology, 71, 616-628.

Montero, I. \& León, 0. (2007). A guide for naming research studies in Psychology. International Journal of Clinical and Health Psychology, 7, 847-862.

Muñoz Umaña, C. (2007). Perspectiva psicológica del bienestar subjetivo. Psicogente, 10(18), 163-173.

Pavot, W. \& Diener, E. (2009). Review of the satisfaction with life scale. En E. Diener (Eds.) Assessing well-being. The collected works of Ed Diener (pp. 101-117). Nueva York: Springer (Social Indicators Research Series, 39).

Peñaguirre Cano, M. \& Rodríguez Mendoza, T. (2017). Un camino hacia el bienestar subjetivo. International Journal of Developmental and Educational Psychology, 2(1), 301-305.

Pérez, G., Anta, C., Badera, S., García, J., Pérez, M. \& Sarrate, M. (2003). Análisis Bibliométrico en Educación. Incidencia en la calidad universitaria. Madrid: Ministerio de Educación y Ciencia de España.

Programa de las Naciones Unidas para el Desarrollo. (2012). Bienestar subjetivo: el desafío de repensar el desarrollo. Santiago: Autor.

Rodríguez-Fernández, A. \& Goni-Grandmontagne, A. (2011). La estructura tridimensional del bienestar subjetivo. Anales de Psicología, 27(2), 327-332.

Rojas, M. (2011). Más allá del ingreso: progreso y bienestar subjetivo. En: M. Rojas (Coord.), La medición del progreso y del bienestar. Propuestas desde América Latina (pp. 29-39). Foro Consultivo Científico y Tecnológico, AC México.

Rueda-Clausen, C., Villaroel-Gutiérrez, C. \& Rueda-Clausen, C. (2005). Indicadores bibliométricos. Origen, aplicación contradicción y nuevas propuestas. Med UNAB, 8(1), 29-36.

Seligman, M. \& Csikszentmihalyi, M. (2000). Positive psychology: An introduction. American Psychologist, 55, $5-14$.

Seligson, J., Huebner, E. \& Valois, R. (2005). An investigation of a Brief Life Satisfaction Scale with Elementary School children. Social Indicators Research, 73(3), 355-374. 


\section{ANÁLISIS BIBLIOMÉTRICO SOBRE EL BIENESTAR SUBJETIVO}

Vázquez, C., Duque, A. \& Hervas, G. (2013). Satisfaction with life scale in a representative sample of Spanish adults: Validation and normative data. Spanish Journal of Psychology. 16(82), 1-15. 